\title{
Evanescent vaso-occlusive choroidal pseudo-tumor with acute painful onset: a presumed vortex vein occlusion
}

\author{
Irmela Mantel • Ann Schalenbourg • Leonidas Zografos
}

Received: 24 July 2013 /Revised: 19 November 2013 / Accepted: 25 November 2013 / Published online: 21 December 2013

(C) Springer-Verlag Berlin Heidelberg 2013

\begin{abstract}
Purpose The aim of our study was to describe the clinical presentation of an unusual evanescent, exudative, choroidal pseudo-tumor with acute painful onset, and propose a pathogenesis.

Methods We carried out a retrospective, observational study using the case series of three patients presenting with an evanescent, exudative, choroidal pseudo-tumor with acute painful onset. Ultra-widefield fluorescein and indocyanine green angiography (ICGA) using the Heidelberg Retina Angiograph and the Staurenghi 230 SLO Retina Lens were used to propose a pathogenesis of this unusual entity.

Results In all three cases, acute ocular pain led to discovery of an exudative, partially hemorrhagic choroidal mass (thickness $2.4 \mathrm{~mm}-4.1 \mathrm{~mm}$ on ultrasound) that quickly regressed within weeks. In the subacute phase, all patients showed choroidal circulation abnormalities on dynamic wide-field ICGA in the affected quadrant, with delayed arterio-venous filling in two patients, and a poorly-defined vortex vein in the third. The choroidal circulation abnormalities resolved within 8 12 weeks, simultaneously with the spontaneous resolution of the choroidal pseudo-tumor. The findings evoked a selfresolving vortex vein occlusion in the corresponding quadrants with acute, painful choroidal exudation.

Conclusions An evanescent, exudative, hemorragic choroidal pseudo-tumor with acute painful onset may be caused by a
\end{abstract}

This study has been presented in parts at the Macula Society 2013, Dana Point, USA

I. Mantel $\cdot$ A. Schalenbourg $\cdot$ L. Zografos

Department of Ophthalmology, University of Lausanne, Jules Gonin

Eye Hospital, Fondation Asile des Aveugles, Lausanne, Switzerland

I. Mantel $(\bowtie)$

University Eye Hospital Jules Gonin, 15 Av de France,

Case postale 133, 1000 Lausanne 7, Switzerland

e-mail: irmela.mantel@fa2.ch vortex vein occlusion. Future patients need to be studied with ICGA in the acute phase to confirm this hypothesis.

Keywords Choroidal pseudo-tumor - Vortex vein occlusion · Choroidal ischemia $\cdot$ Ocular pain $\cdot$ Dynamic ultra-widefield angiography

\section{Introduction}

Choroidal mass lesions most often correspond to a choroidal neoplasm, but occasionally they represent acellular, exudative and/or hemorrhagic choroidal lesions, also called "choroidal pseudo-tumors" $[1,2]$. The most common form of these exudative choroidal pseudo-tumors is known as peripheral, exudative, hemorrhagic chorioretinopathy, [1-6] characterized by painless pigment epithelium detachments with hemorrhages and/or lipid exudates in the peripheral fundus of aged patients. Recently, we reported polypoidal, choroidal vascular abnormalities in association with these lesions [4].

However, we have seen a few patients with transient choroidal pseudo-tumors whose clinical presentation did not match that of a peripheral, exudative, hemorrhagic chorioretinopathy: these patients were younger, presented with acute ocular pain, and their choroidal mass lesion was not associated with lipid exudates, all the while regressing rapidly into a subtle scar.

A wide-field contact lens system for the scanning laser ophthalmoscope (SLO), which was developed by Staurenghi et al., provides a five-fold increase of the photographic angle up to $150^{\circ}$, thereby allowing an easier visualization of the peripheral fundus and dynamic imaging of the early angiographic phase [7]. Since October 2007, new patients with a fundus tumor referred to the oncology unit were systematically examined with this technique. On the basis of the dynamic ultra-widefield angiographic images with fluorescein and 
indocyanine green, we propose a pathogenesis for this unusual, painful, and transient choroidal pseudo-tumor.

\section{Methods}

This was a retrospective chart study of three consecutive patients with painful, transient choroidal pseudo-tumors referred to the Ocular Oncology Unit of the Jules-Gonin Eye Hospital (Lausanne, CH), since October 2007. Ethics committee approval was obtained. The study adhered to the tenets of the Declaration of Helsinki.

All patients had a complete systemic and ocular history taken and had undergone a complete ophthalmic examination, including best corrected visual acuity (BCVA), slit-lamp examination, intraocular pressure (IOP), and dilated fundus examination. Panoramic fundus color photos were taken with the Panoret ${ }^{\circledR} 1000$ M Camera (Medibel/ CMT Technology, Haifa, Israel), using its $100^{\circ}$ optic contact lens and transscleral illumination. B-scan ultrasonography was performed by a retinal specialist with the Quantel Medical CineScan (Version S 5.06; Quantel Medical, Clermont-Fernand, France) using both the 10 and $20 \mathrm{MHz}$ probe.

Patients underwent ultra-widefield angiography of both eyes, with dynamic, early-phase imaging in the affected eye, using the Heidelberg Retina Angiograph (HRA 2; Heidelberg Engineering $\mathrm{GmbH}$, Heidelberg, Germany) and the Staurenghi 230 SLO Retina Lens (Ocular Instruments, Inc., Bellevue, Wa, USA) for ultra-widefield fluorescein angiography (FA) and indocyanine green angiography (ICGA) with panoramic images up to $150^{\circ}$. The dynamic sequence was started as soon as the dye reached the eye (about 10 seconds after IV injection), with the HRA2 capturing 8.8 frames/ second during 30 to 40 seconds. The images of the two seconds before its manual start were automatically included in the film. Late images were taken up to 30 minutes after the dye injection. All images were taken with the high-speed mode (image resolution $768 \times 768$ pixels).

\section{Results}

Case reports

\section{Patient 1}

In November 2008, a 65-year-old white male with a general history of treated arterial hypertension, hypercholesteremia, and depression presented with acute, severe pain in his left eye (LE). His BCVA was 0.8 . He had an elevated IOP of $50 \mathrm{mmHg}$ and was treated with miotic drops (LE) and a Mannitol IV infusion, resulting in normalization of his IOP. Fundus examination revealed a choroidal mass in the temporal inferior quadrant of the same eye. B-scan ultrasonography measured a thickness of $2.4 \mathrm{~mm}$ (Fig. 1a). The patient was referred to our ocular oncology unit.

Two weeks later, on examination in our ocular oncology unit, BCVA was 1.0 in both eyes, with an IOP of $17 \mathrm{mmHg}$ (RE) and $13 \mathrm{mmHg}(\mathrm{LE})$. The anterior segments were normal, with an open iridocorneal angle and a clearly visible scleral spur on gonioscopy. An iridocorneal angle of $51^{\circ}$ (LE) was measured with the anterior segment OCT (Visante AS OCT, Carl Zeiss Meditec, IUC, Dublin, CA, USA). A dilated fundus examination and B-scan ultrasonography only revealed a flat hemorrhagic lesion inferior to the temporal inferior vascular arcade (Fig. 1b,c). Ultra-widefield FA showed a normal retinal perfusion. However, on ICGA the choroidal arteries of the temporal inferior quadrant started to fill $1 \mathrm{~s}$ after the choroidal arteries in the other quadrants (Fig. 1d). The venous filling on ICGA was also comparatively reduced, with a poor filling of the temporal inferior vortex vein. The presumed diagnosis was that of a transient, hemorrhagic choroidal pseudo-tumor of choroidal vascular origin.

\section{Patient 2}

In January 2009, a 30-year-old white male in good general health suffered from right orbital pain, for which he consulted ten days later. His ophthalmologist found a BCVA of 0.9 , and an ipsilateral, temporally restricted visual field, corresponding to a large, nasal, parapapillary and yellowish choroidal mass (Fig. 2a), with a thickness of $2.9 \mathrm{~mm}$ on B-scan ultrasonography (Fig. 2b). Intraocular pressure was $18 \mathrm{mmHg}$, and a systemic work-up (hematology, blood pressure, brain CT-scan and MRI, and carotid Doppler ultrasonography) was normal. The patient was referred with the diagnosis of a 'choroidal mass of unknown origin'.

Five weeks later, upon examination in our ocular oncology unit, BCVA was 1.0 in both eyes. The anterior segments were normal, and the right fundus showed only a pale, parapapillary, residual choroidal lesion, of $1.5 \mathrm{~mm}$ in thickness on B-scan ultrasonography (Fig. 2c,d). Ultra-widefield FA showed a normal retinal perfusion, as well as some speckled RPE hyper-/hypofluorescence in the nasal area (images not shown). Dynamic, ultra-widefield ICGA revealed a clearly delayed arterial choroidal filling of the nasal superior quadrant, as well as a mild delay in the nasal inferior quadrant (Fig. 2e). The venous filling remained reduced in the nasal superior quadrant during the whole angiographic sequence, and was associated with a slim vortex vein (Fig. 2f). A choroidal vascular origin was suspected as the cause of a regressive, exudative choroidal pseudo-tumor.

Two months later, the mildly yellowish lesion had completely flattened (Fig. 2g). On ICGA this time, the nasal-arterial choroidal filling delay was less pronounced 

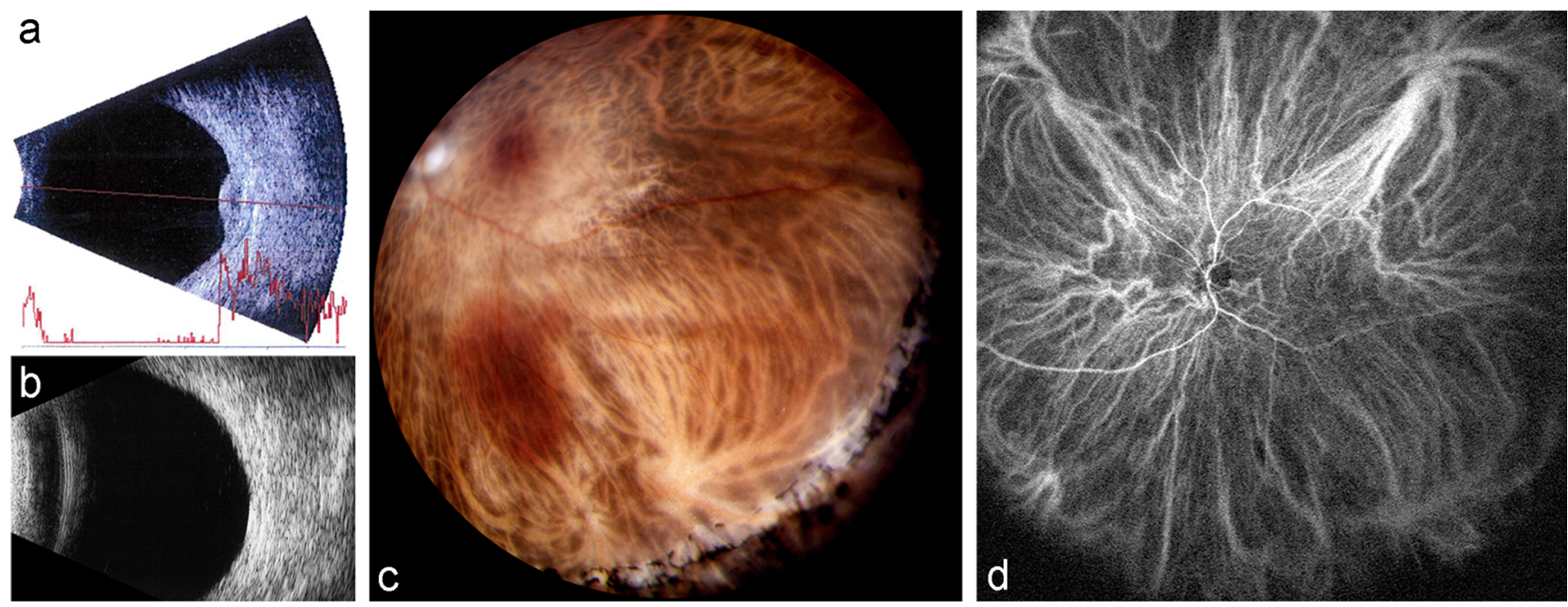

Fig. 1 a B-scan ultrasonography of patient 1 at first visit revealed a choroidal mass $($ Height $=2.4 \mathrm{~mm})$ in his left eye. b On referral 2 weeks later, ultrasonography revealed lesion regression, and $\mathbf{c}$ fundus color photography showed a flat, slightly hemorrhagic lesion inferior to the temporal inferior vascular arcade. d At this time point, ultra-widefield

(0.7 seconds), and the venous filling was poorer in the nasal than in the temporal quadrants (Fig. 2h, i).

\section{Patient 3}

In September 2011, a 64-year-old white male with a history of moderate, pre-capillary pulmonary hypertension and ischemic heart disease suffered from acute, severe left-orbital pain. Upon examination, his BCVA was 1.0, and his ophthalmologist discovered a choroidal mass in the temporal inferior quadrant (LE), which he documented with FA, ICGA, and B-scan ultrasonography (thickness $4.1 \mathrm{~mm}$ ) (Fig. 3a, b, c). IOP was $21 \mathrm{mmHg}$. The patient was referred with suspicion of choroidal melanoma.

Two weeks later, the patient presented in our ocular oncology unit without pain. Vision was full in both eyes, the anterior segments were normal, and IOP was $21 \mathrm{mmHg}$ in both eyes. The peripheral, temporal inferior, whitish mass lesion (LE) had significantly reduced in thickness $(2.1 \mathrm{~mm}$ on ultrasonography, Fig. 3d, e). The retinal perfusion was normal on ultra-widefield FA. On ultra-widefield ICGA, the choroidal arterial filling appeared simultaneously in all quadrants. However, in the venous phase, the temporal inferior vortex vein was poorly-demarcated, filling poorly (Fig. 3f). Consequently, a diagnosis of a pseudo-tumor in partial, spontaneous regression and of choroidal vascular origin was presumed.

On a follow-up visit 3 months later, the mass had completely disappeared (Fig. 3g) and BCVA was unchanged at 1.0. Dynamic ultra-widefield FA and ICGA were normal, including a good visibility of two distinct, temporal inferior vortex veins (Fig. 3h). indocyanine green angiography is shown $23 \mathrm{~s}$ after arrival of the dye in the choroidal circulation: temporal inferior quadrant with a poorly-filling, ill-defined vortex vein. The dynamic sequence showed an arterial filling of the same quadrant, which was delayed by $1 \mathrm{~s}$ as compared to the other quadrants, with subsequently reduced venous filling

\section{Summary of the cases}

In summary, these three white, male patients with a mean/ median age of 53/64 years, respectively, shared the following clinical characteristics: they had suffered from acute ocular pain that motivated an ophthalmic visit, during which a choroidal mass was discovered (and in one case, an elevated IOP). Upon referral to our ocular oncology unit 2-5 weeks later, dynamic ultra-widefield ICGA revealed a disturbed choroidal circulation in the corresponding quadrants, though the exudative hemorrhagic mass itself was found to be in partial or even complete regression, leaving only a subtle, flat chorioretinal scar. When we speculated about the origin of these transient, painful choroidal pseudo-tumors, the ICGA findings pointed toward the choroidal circulation: both the quadrant-wise choroidal circulation abnormality and the acute, hemorrhagic exudative choroidal mass may be secondary to an acute, self-resolving vortex vein occlusion.

\section{Discussion}

In this report, we describe the clinical and angiographic observations in three patients with an acute painful, evanescent, unilateral choroidal pseudo-tumor, partially hemorrhagic, that rapidly regresses into a subtle chorioretinal scar. Because of the painful onset, patients were rapidly referred to our ocular oncology unit, allowing a detailed examination in the subacute phase (after 2-5 weeks). Nevertheless, compared to the initial examination results in the referral charts, all patients showed a spontaneous regression of the mass lesion at the time we examined them. Because of this rapidly transient character 

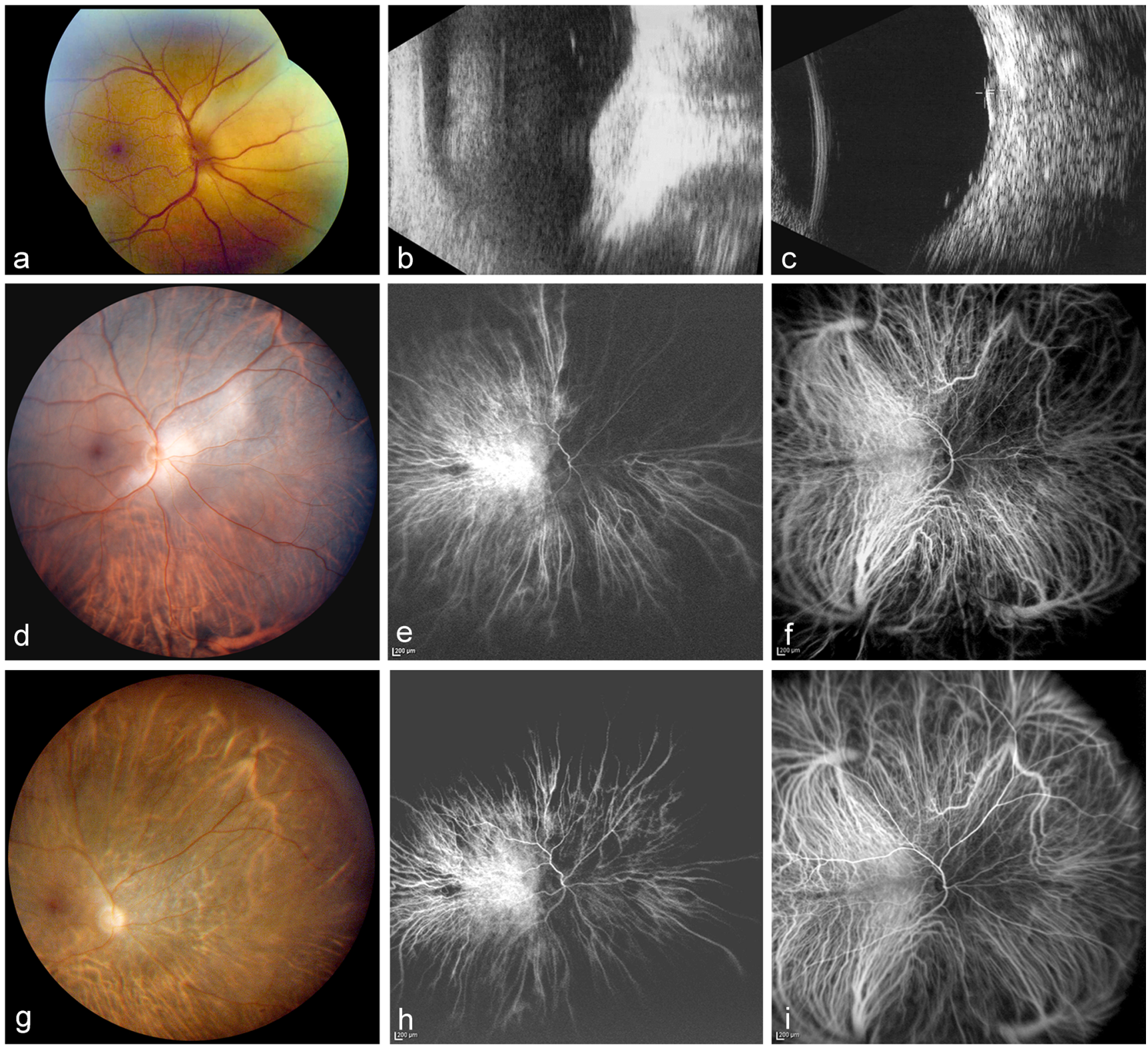

Fig. 2 a Fundus color photography of patient 2 at his first ophthalmic examination documented a yellowish choroidal mass supero-nasally to the disc in his right eye. b B-scan ultrasonography measured $2.9 \mathrm{~mm}$ thickness. c On referral 5 weeks later, the choroidal lesion had regressed to $1.5 \mathrm{~mm}$ thickness on B-scan ultrasonography. d Fundus color photography showed partial regression. e Ultra-widefield indocyanine green angiography $3.0 \mathrm{~s}$ after arrival of the dye in the choroidal circulation: severely delayed choroidal arterial filling of the nasal superior quadrant and mild arterial hypoperfusion of the nasal inferior quadrant. The dynamic sequence allowed measuring a 2.0-s filling delay in the nasal superior and a 0.8 -s delay in the nasal inferior quadrant. f Ultra-widefield

of the exudative mass lesion, which was similar to the time course in the multiple evanescent white dot syndrome, we chose the descriptive term "evanescent" as most appropriate to describe this hallmark of the disorder. However, due to this evanescent time course, our dynamic ultra-widefield angiographic results may not fully show the initial characteristics of this pseudo-tumor. indocyanine green angiography $10 \mathrm{~s}$ after arrival of the dye: reduced venous filling in the nasal superior quadrant with a slim vortex vein ampulla. g After 2 months of follow-up, fundus color photography showed a flat, slightly yellowish residual lesion. h Ultra-widefield indocyanine green angiography $2.7 \mathrm{~s}$ after arrival of the dye in the choroidal circulation: mild arterial hypoperfusion in the nasal quadrants. The dynamic sequence identified a $0.7 \mathrm{~s}$ delay in both nasal quadrants. i Ultrawidefield indocyanine green angiography $34 \mathrm{~s}$ after arrival of the dye: less pronounced venous filling in the nasal as compared to the temporal quadrants

Despite this examination delay, dynamic ultra-widefield ICGA showed some obvious abnormalities of the choroidal circulation, characterized by a delayed arterio-venous choroidal filling and/or poor filling of the vortex vein in the quadrant of the exudative pseudo-tumor, compared to the unaffected areas in the same eye. Though the anatomy of the choroid is highly variable, a delayed arterio-venous filling of one (or 

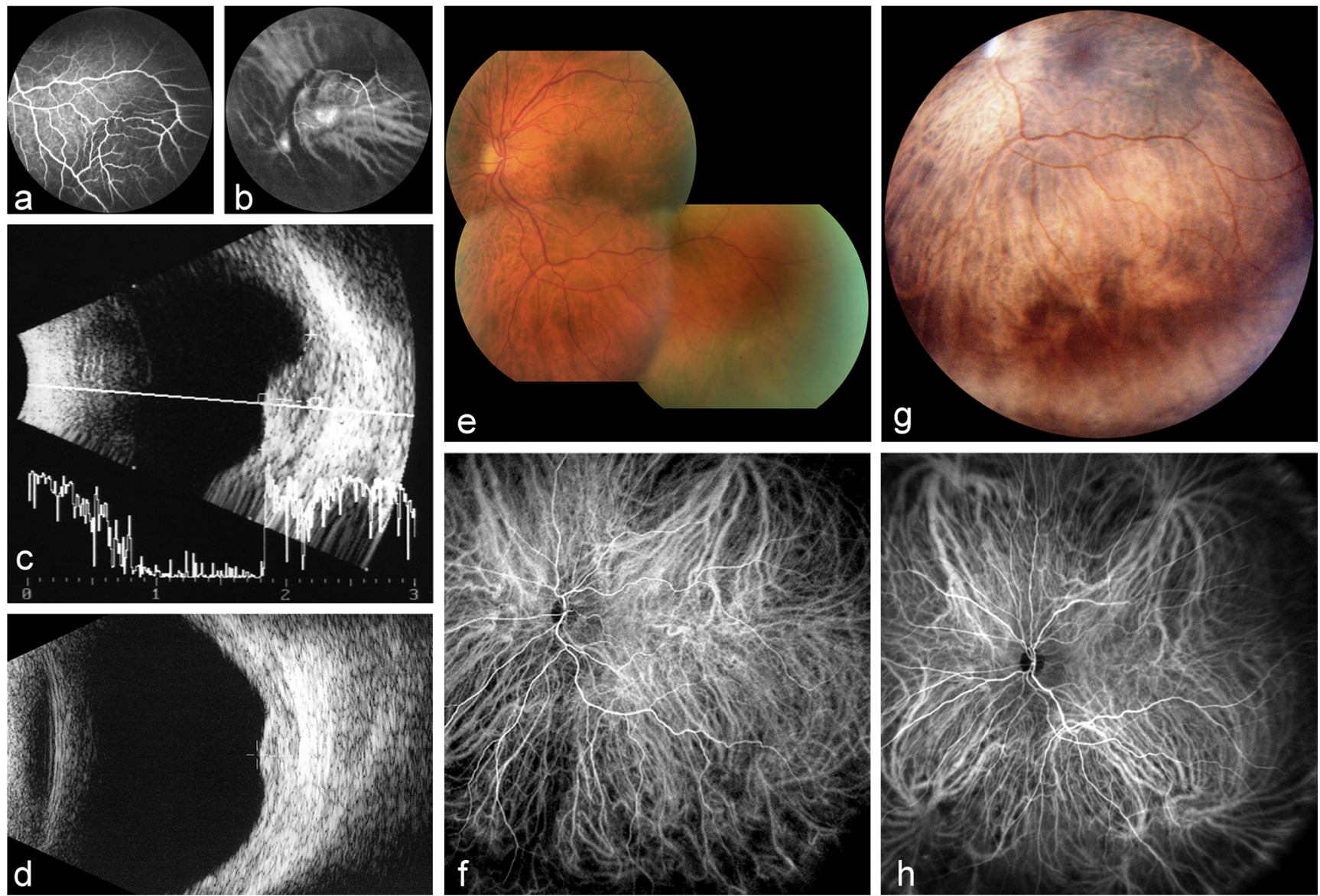

Fig. 3 a, b Patient 3 at his first ophthalmic examination: peripheral fluorescein (Fig. 3a) and indocyanine green (Fig. 3b) angiography showed an elevation of the retinal and choroidal vasculature, with a non-specific hyperfluorescence on indocyanine green angiography. $\mathbf{c}$ Bscan ultrasonography measured a thickness of $4.1 \mathrm{~mm}$. d On referral 2 weeks later, B-scan ultrasonography revealed partial regression to 2.1$\mathrm{mm}$ thickness. e Color fundus photography showed the temporal inferior

two) of its quadrants is clearly pathologic. The localization of these circulation abnormalities in the same quadrant as the exudative pseudo-tumor was suggestive to us of their pathogenic role in this disorder.

Checking the literature, we found several experimental studies showing that both an arterial and venous choroidal obstruction can induce exudative changes in the subretinal space, associated with retinal pigment epithelium (RPE) alterations [8-11]. Additionally, both arterial[9] and venous [10-13] choroidal occlusions have been reported to provoke a delayed filling of the choroidal circulation, clearly visible on ICGA [14]. The distribution of the affected area depends on the affected vessel. While the zone of irrigation from the posterior ciliary arteries is typically triangular, from the macular area enlarging towards the periphery $[9,10,15,16]$, the vortex veins each drain their quadrant of the choroid [11-13]. Thus, the choroidal circulation abnormality found in our patient with a quadrant-wise distribution of delayed arteriovenous filling, ill-defined vortex vein ampullae, and a choroidal mass. f Ultra-widefield indocyanine green angiography $7.4 \mathrm{~s}$ after arrival of the dye in the choroidal circulation, revealing an ill-defined temporal inferior vortex vein with poor filling. $g$ After 3 months of follow-up, fundus color photography of the left eye showed no mass anymore. $\mathbf{h}$ On ultra-widefield indocyanine green angiography $31 \mathrm{~s}$ after arrival of the dye, the temporal inferior vortex vein had reappeared as two distinct ampullae, with a normalized venous choroidal filling

transient choroidal mass in the same quadrant, is compatible with a vortex vein occlusion, but not with an arterial choroidal obstruction. Such transient shutting of the vortex vein may well be responsible for an exudative, and sometimes hemorrhagic mass lesion of the choroid in the same quadrant, with a dome-shaped, elevated pigment epithelium.

In the clinical literature, we found one study about a vortex vein occlusion following scleral buckling with cryopexy for retinal detachment [17]. They found a choroidal detachment in the early phase ( 2 weeks) associated with venous congestion, followed by a remodeling of the venous choroidal circulation and disappearance of the vortex vein ampullae after several months to years [17]. Similarly, acute venous pressure rise during the Valsalva maneuver may cause suprachoroidal hemorrhage [18, 19]. Interestingly, suprachoroidal hemorrhage has also been described to occur spontaneously, associated with high myopia and aspirin [18].

Looking for an explanation for the pain, we found that vortex vein occlusion has been reported to affect the anterior 
segment as well, provoking exudative changes within the ciliary body and iris $[10,13]$, and potentially leading to increased intraocular pressure and pain. Experimentally, the occlusion of at least three vortex veins in monkeys induced an IOP rise, with a shallow anterior chamber and iris hyperemia, resolving completely within two weeks [13]. Though in this animal experiment, the occlusion of only one to two vortex veins was insufficient to produce an IOP rise, it did provoke anterior chamber exudation [13]. We speculate that in our series, the one patient with severe IOP rise in the acute phase experienced transient closure of his iridocorneal angle, related to a widespread, anterior segment venous congestion. The fact that all our patients suffered an acute onset, transient pain could be attributed to the ischemia and/or inflammation associated with a vortex vein occlusion. However, the acute exudative choroidal mass itself may cause stress on the sclera, and therefore cause acute pain, similar to the reports of suprachoroidal hemorrhage causing a tender eye $[18,19]$.

The evanescent character with rapid, spontaneous resolution of these choroidal pseudo-tumors also corresponds with the rapid normalization of the choroidal circulation observed in experimental vortex vein occlusion [13]. We mentioned that our angiographic images may not show the full extent of the initial occlusion. The rapid resolution could explain why patient 3 did not show any filling delay, but only a poorly filling and ill-demarcated vortex vein in the corresponding quadrant. Furthermore, such partial resolution may explain the absence of a venous engorgement, which we would normally expect.

Finally, the scars after resolution of these pseudo-tumors were discrete, without the typical speckled pigmentary changes following an arterial choroidal infarction [9]. Experimental vortex vein occlusions have been demonstrated to result in relatively few choroidal changes [8-10] compared to the triangular pigment alterations after a posterior ciliary artery occlusion $[9,16]$. It is interesting to note that a simultaneous vortex vein occlusion has a protective effect in animals with an arterial choroidal infarction, resulting in less severe chorioretinal changes than in those with an isolated arterial occlusion $[20,21]$. Anecdotally, this has been used as innovative therapeutic approach for age-related macular degeneration with RPE detachment, based on its putatively ischemic choroidal origin $[22,23]$.

The present study is limited by its small number of patients. As it is retrospective, an extensive work-up for systemic, vasoocclusive risk factors is unavailable, though two patients had experienced previous cardiovascular problems. However, it is interesting that two out of three patients had arterial hypertension, which is a common association in choroidal pseudotumors. The suspected cardiovascular risk profile gives further support to the vascular hypothesis of this disorder.

In conclusion, the cases presented in this study, of choroidal pseudo-tumors with acute onset pain, a delayed choroidal circulation, and ill-defined vortex veins in the corresponding quadrant as well as rapid, spontaneous resolution, evoke the hypothesis of a transient vortex vein occlusion. To the best of our knowledge, this is the first report documenting spontaneous transient choroidal pseudo-tumors in association with signs of a vortex vein occlusion. Future patients with this uncommon clinical presentation need to be examined in the acute phase with dynamic ultra-widefield ICGA in order to better characterize this condition. Furthermore, a systemic cardiovascular work-up of patients with a suspected vortex vein occlusion is needed, in order to better define their risk profile.

\section{Financial Support None}

Conflict of interest The authors declare that they have no conflicts of interest.

\section{References}

1. Shields CL, Salazar PF, Mashayekhi A, Shields JA (2009) Peripheral exudative hemorrhagic chorioretinopathy simulating choroidal melanoma in 173 eyes. Ophthalmology 116(3):529-535

2. Shields JA, Mashayekhi A, Ra S, Shields CL (2005) Pseudomelanomas of the posterior uveal tract: the 2006 Taylor R. Smith Lecture. Retina 25(6):767-771

3. Annesley WH Jr (1980) Peripheral exudative hemorrhagic chorioretinopathy. Trans Am Ophthalmol Soc 78:321-364

4. Mantel I, Schalenbourg A, Zografos L (2012) Peripheral exudative hemorrhagic chorioretinopathy: polypoidal choroidal vasculopathy and hemodynamic modifications. Am J Ophthalmology 153(5):910 922

5. Mantel I, Uffer S, Zografos L (2009) Peripheral exudative hemorrhagic chorioretinopathy: a clinical, angiographic, and histologic study. Am J Ophthalmol 148(6):932-938

6. Silva VB, Brockhurst RJ (1976) Hemorrhagic detachment of the peripheral retinal pigment epithelium. Arch Ophthalmol 94(8): 1295-1300

7. Staurenghi G, Viola F, Mainster MA, Graham RD, Harrington PG (2005) Scanning laser ophthalmoscopy and angiography with a wide-field contact lens system. Arch Ophthalmol 123(2):244-252

8. Algvere P (1976) Retinal detachment and pathology following experimental embolization of choroidal and retinal circulation. Graefes Arch Clin Exp Ophthalmol 201(2):123-134

9. De Laey JJ (1983) Fluorescein angiography of the choroid in health and disease. Int Ophthalmol 6(2):125-138

10. Gaudric A, Coscas G, Bird AC (1982) Choroidal ischemia. Am J Ophthalmology 94(4):489-498

11. Gaudric A, Sterkers M, Coscas G (1987) Retinal detachment after choroidal ischemia. Am J Ophthalmology 104(4):364-372

12. Nishikawa M, Matsunaga H, Takahashi K, Matsumura M (2009) Indocyanine green angiography in experimental choroidal circulatory disturbance. Ophthalmic Res 41(1):53-58

13. Hayreh SS, Baines JA (1973) Occlusion of the vortex veins. An experimental study. Br J Ophthalmol 57(4):217-238

14. Bischoff PM, Speiser $P$ (1986) Angiographic study of the vortex vein circulation. Graefes Arch Clin Exp Ophthalmol 224(2):122-123

15. Amalric P, Biau C, Cenac P (1969) Choroid changes of a triangular type. Bull Mem Soc Fr Ophtalmol 82:449-455 
16. Amalric P (1971) Acute choroidal ischaemia. Trans Ophthalmol Soc U K 91:305-322

17. Takahashi K, Kishi S (2000) Remodeling of choroidal venous drainage after vortex vein occlusion following scleral buckling for retinal detachment. Am J Ophthalmology 129(2):191-198

18. Chak M, Williamson TH (2003) Spontaneous suprachoroidal haemorrhage associated with high myopia and aspirin. Eye 17(4):525-527

19. Hammam T, Madhavan C (2003) Spontaneous suprachoroidal haemorrhage following a valsalva manoeuvre. Eye 17(2):261262
20. Hayreh SS, Chopdar A (1982) Occlusion of the posterior ciliary artery. V. Protective influence of simultaneous vortex vein occlusion. Arch Ophthalmol 100(9):1481-1491

21. Loeffler KU, Hayreh SS, Tso MO (1994) The effects of simultaneous occlusion of the posterior ciliary artery and vortex veins. A histopathologic study. Arch Ophthalmol 112(5):674-682

22. Bonnet M (1984) Surgical occlusion of 2 vorticose veins in the treatment of decompensated senile macular degeneration. J Fr Ophtalmol 7(8-9):563-567

23. Bonnet M (1985) Surgical occlusion of 2 vortex veins in the treatment of decompensated senile macular degeneration. A longer-term evaluation. J Fr Ophtalmol 8(12):779-783 